\title{
Upstream Therapy for Atrial Fibrillation Prevention: The Role of Sacubitril/Valsartan
}

\author{
Renato De Vecchis ${ }^{\mathrm{a}, \mathrm{d}}$, Andrea Paccone ${ }^{\mathrm{b}}$, Marco Di Maio ${ }^{\mathrm{c}}$
}

\begin{abstract}
The therapy or prevention of atrial fibrillation (AF) is defined as upstream therapy when conducted with the use of drugs, e.g., angiotensin-converting enzyme inhibitors (ACEIs), angiotensin receptor antagonists, statins, and omega- 3 fatty acids, not included in the classes of antiarrhythmic drugs recognized by the Vaughan Williams classification. In our review, we illustrate the rational bases of upstream AF therapy, which encompasses drugs having the property to reduce hemodynamic congestion and cardiac overload, as in the case of ACEIs or angiotensin receptor blockers, as well as drugs able to prevent atrial fibrosis or reduce oxidative stress, such as statins or omega-3 fatty acids, respectively. In this review, randomized controlled trials (RCTs) conducted with the abovementioned drugs are examined. Really, these RCTs have generated mixed results. In the context of the prevention and therapy of AF, our experience is then presented, relating to a patient with heart failure and reduced left ventricular ejection fraction, with a history of relapsing episodes of paroxysmal AF. In this patient, administration of sacubitril/valsartan at appropriate doses allowed recovery of the sinus rhythm. Therefore this case testifies how the upstream therapy of AF might have good results when conducted with sacubitril/valsartan. Thus, RCTs with adequate statistical power are warranted in order to confirm the preliminary encouraging result of our case report, and validate a useful role of sacubitril/valsartan as an upstream therapy of AF.
\end{abstract}

Keywords: Atrial fibrillation; Angiotensin receptor neprilysin inhibitor; Atrial remodeling; Outcome

\section{Upstream Treatment With Non-Antiarrhythmic Drugs for Atrial Fibrillation (AF)}

In recent years, considering the uninspiring results of classic

Manuscript submitted April 17, 2020, accepted April 27, 2020

Published online June 3, 2020

aMedical and Polyspecialist Centre, DSB 29 "S. Gennaro dei Poveri Hospital", via S.Gennaro dei Poveri 25, 80136 Naples, Italy

bDepartment of Cardiology, University of Bari “Aldo Moro", Bari, Italy

'Department of Cardiology, University of Campania "Luigi Vanvitelli”, 80138

Naples, Italy

${ }^{\mathrm{d} C}$ Corresponding Author: Renato De Vecchis, Medical and Polyspecialist Centre, DSB 29 "S. Gennaro dei Poveri Hospital", via S.Gennaro dei Poveri 25, 80136 Naples, Italy. Email: devecchis.erre@virgilio.it

doi: https://doi.org/10.14740/cr1073 antiarrhythmic drugs, other not strictly antiarrhythmic drugs have been taken into account for the treatment of AF, with the aim of correcting the substrate underlying the arrhythmia and to modify its evolution (upstream treatment), thus preventing both the first episode of AF (primary prevention) and relapses (secondary prevention). It is known that $\mathrm{AF}$ in itself determines electrical modifications $[1,2]$ and structural changes of the atria [1-4] represented essentially by a shortening and dispersion of the action potential, atrial enlargement and fibrosis (so called remodeling), which then make the restoration of the sinus rhythm and its subsequent maintenance more problematic. The upstream treatment aims to counter and/or delay this remodeling process by acting at different levels and with different mechanisms, such as the blockade of the renin-angiotensin-aldosterone system, the antifibrotic and anti-inflammatory effect, the reduction of oxidative stress, etc.

According to the most credited thesis, the drugs that can be used for upstream prevention of AF would prevent $\mathrm{AF}$ through an improvement of systemic and pulmonary hemodynamics, as in the case of angiotensin-converting enzyme inhibitors (ACEIs) and sartans, or through a pleiotropic effect of prevention of atrial fibrosis, such as in the case of statins, or through a reduction in oxidative stress, as postulated for omega- 3 fatty acids.

Information is provided below regarding the upstream treatment of AF with each of these drug classes.

\section{ACEIs and angiotensin II receptor antagonists (ARBs)}

Atrial stretch and inflammation have been shown to increase the level of angiotensin II in the heart. Angiotensin II in turn causes calcium overload and atrial fibrosis, two essential conditions for electrophysiological changes (shortening of refractoriness and slowing of conduction), which favor the initiation and maintenance of AF. Some experimental studies have shown that both ACEIs [5] and ARBs [6, 7] are able to prevent the electrical and mechanical remodeling that is observed in AF.

Overall the published data, even if mainly consisting of post hoc analyses of studies with endpoints different from the occurrence of AF, seem to support the role of ACEIs and ARBs in primary AF prevention, at least in patients with heart failure [8]. Regarding secondary AF prevention (prevention of recurrence in patients with previous episodes of arrhythmia) initially some small prospective studies were published, in which the treatment with only the ACEI [9], or the association 
of ACEIs or ARBs with chronic antiarrhythmic treatment with amiodarone resulted in a statistically significant reduction in recurrence of AF [10,11].

However, subsequently, three large prospective studies, randomized with ARB vs. placebo, have instead highlighted an absence of effect of these drugs in preventing AF recurrences in various clinical settings. The GISSI-AF [12], which enrolled 1,442 patients, mainly with underlying heart disease and affected by arterial hypertension, has not shown any efficacy of valsartan in reducing the number of arrhythmic recurrences in a 12-month follow-up. In the valsartan group, 51.4\% of patients had at least one AF recurrence vs. $52.1 \%$ of the placebo group.

In addition, in the Active study [13] in a population of 1,730 patients with characteristics similar to those of GISSI$\mathrm{AF}$, irbesartan did not reduce the AF recurrence rate at a 4.1year follow-up. Finally, in ANTIPAF [14] 425 patients with paroxysmal AF were studied in the absence of structural heart disease at a 12-month follow-up, and olmesartan did not determine any modification of the AF burden.

Based on the above results, it appears that ACEIs and ARBs can modify the underlying heart disease, and in this way reduce the incidence of new-onset episodes of AF (primary prevention) at least in patients with left ventricular dysfunction and/or heart failure. However today, based on a recent metaanalysis [15], the use of ACEIs and ARBs appears not justified in the secondary prevention of AF recurrences, at least outside of what are the other indications for the use of these drugs. It remains to be investigated the possible clinical efficacy in the prevention of AF with aldosterone antagonists, which have an important antifibrotic effect.

\section{Statins}

Statins, in addition to reducing cholesterolemia, have some pleiotropic effects that can act favorably on the substrate of $\mathrm{AF}$ and on atrial remodeling, such as an anti-inflammatory and antioxidant action [16]. In addition, increasing the synthesis of nitric oxide at the endothelial level can play a protective effect on the extracellular matrix. In fact, in some experimental studies, statins have been shown to reduce inflammatory processes and formation of fibrous tissue in the atria, to prevent shortening of refractory periods and slowing-down of conduction and to reduce the duration of AF accesses compared to controls $[17,18]$.

In the clinic, there are some retrospective and prospective studies on the clinical efficacy of statins in the primary and secondary prevention of AF [19-21]. The data are conflicting and obtained mainly from retrospective studies while the prospective studies are small. Given the anti-inflammatory action of statins, their effect on the prevention of AF in cardiac surgery has been studied in particular. The ARMYDA-360 study has highlighted a significant reduction in the incidence of postoperative episodes of AF in 200 patients randomized to atorvastatin vs. placebo. Although the data are not always confirmed in subsequent studies, in the meta-analysis by Chen et al [22] there is a statistically significant benefit of statins in the prevention of AF in cardiac surgery. Instead, in other clini- cal situations of primary and secondary prevention, including studies after atrial ablation [23], statins are not proved effective in preventing AF [24].

\section{Omega-3 fatty acids}

The data regarding atrial arrhythmias are limited. The first observational study, involving 4,815 subjects aged $>65$ years with a follow-up of 12 years [25], showed a reduction of 28$31 \%$ of the incidence of AF among subjects with regular fish intake in the diet. These data, however, were not confirmed in two large subsequent studies [26, 27].

Recently three prospective randomized studies centered on the prevention of recurrence of AF have given conflicting results. In the study of Nodari et al [28] in 199 patients with persistent $\mathrm{AF}$, in chronic treatment with amiodarone and ACEIs or ARBs, the addition of n-3 polyunsaturated fatty acid (PUFA) resulted in a statistically significant reduction in arrhythmia relapses after electrical cardioversion. On the contrary, the study by Kowey et al [29] in 663 patients with paroxysmal or persistent AF, showed no effect of n-3 PUFAs in reducing the risk of recurrence of AF. Negative results were also obtained in the study by Bianconi et al [30] in 200 patients after cardioversion of AF.

In a recent meta-analysis [31], relating to 1,955 patients, n-3 PUFA were ineffective in both primary prevention (including AF after cardiac surgery), and secondary AF prevention. This data were confirmed in the results of the OPERA study, which assessed the effect of the administration of n-3 PUFAs on the incidence of AF in the first 10 days after surgery in 1,516 patients for coronary or valvular cardiac surgery. In fact, the incidence of AF was substantially similar in the placebo group compared to the group pretreated with n-3 PUFA [32]. Therefore, in light of these results, there are currently insufficient data to propose n-3 PUFA therapy in the prevention of primary or secondary AF, outside of what are the conventional indications for the use of these drugs.

\section{New Horizons for the Upstream Therapy: The Role of Sacubitril/Valsartan}

In the upstream therapy scenario, sacubitril/valsartan enters with a substantially new role. In fact, unlike beta blockers, ACEIs and sartans, this drug is not a neurohormonal blocker, but rather a drug that amplifies and enhances the action of cardiac hormones, namely the atrial natriuretic peptide (ANP) and the B-type natriuretic peptide (BNP), the latter being synthetized by ventricular myocytes in response to increases of wall stretch [33]. Sacubitril/valsartan does not have an intrinsic antiarrhythmic effect; however the increase in the half-life of ANP and BNP that it induces allows these cardioprotective hormones to more intensely regulate the function of the atria and ventricles. This occurs at the level of the atria through a preservation of the reservoir function, that is, the aspiration of caval and pulmonary venous blood into the right and left atrium, respectively, during the ventricular systole [34]. More- 

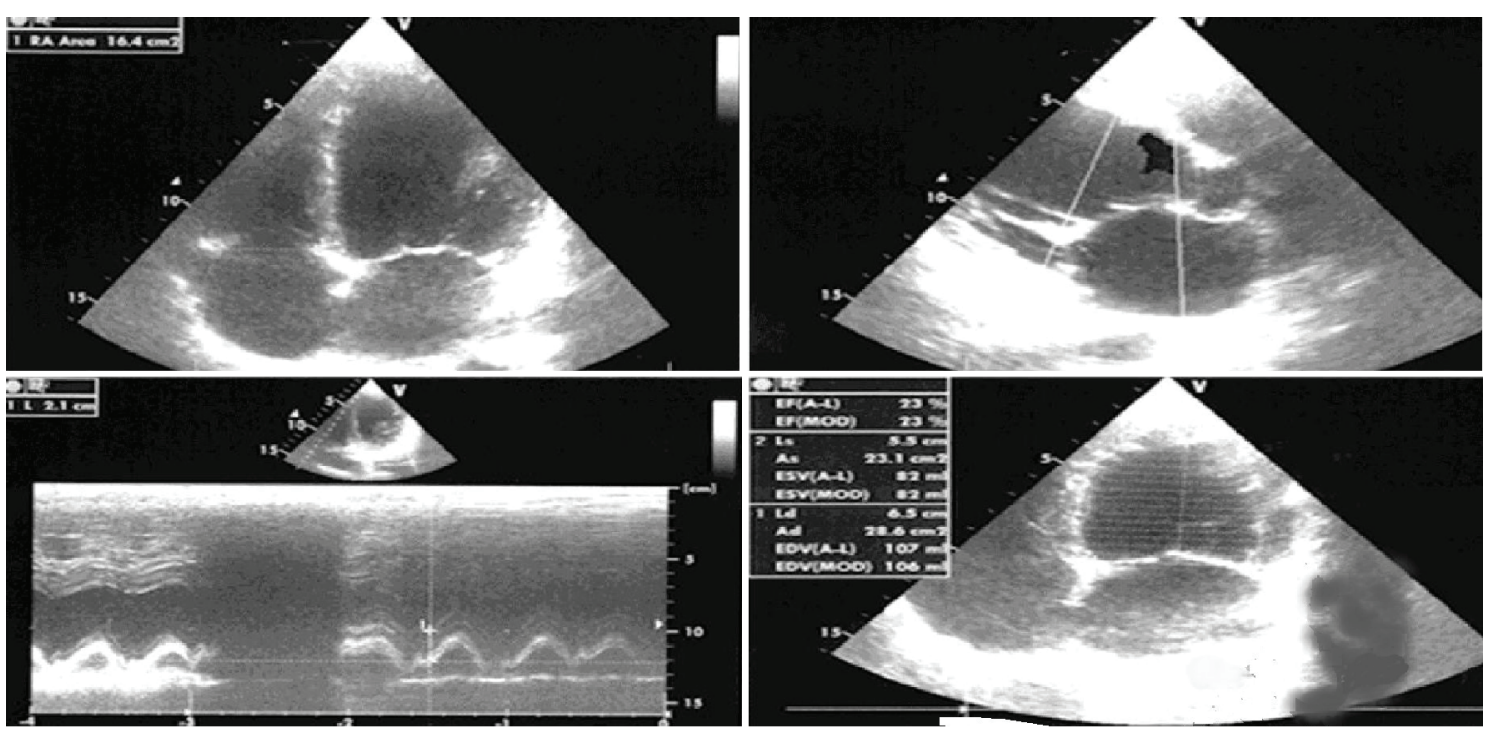

Figure 1. Transthoracic echocardiography (TTE) at the patient's first visit.

over, at the ventricular level, the drug acts by increasing the global longitudinal strain [35]. In other words, it improves the depressed stroke volume by retrieving the contractile efficiency especially along the sagittal-longitudinal axis, rather than by strengthening the wall kinesis in the radial direction [35]. Thus, the restoration of the hemodynamic balance obtained with sacubitril/valsartan is able to regularize the heart rhythm, by inducing a "reverse remodeling" of the atria both morphologic and electroanatomic.

There is little data on the literature regarding a possible role of sacubitril/valsartan for the prevention of AF [36-38]. In fact, there are currently no randomized controlled trials (RCTs) concerning the use of sacubitril/valsartan for antiarrhythmic purposes. Instead, the case report presented below would seem to support the efficacy of sacubitril/valsartan used as upstream therapy for AF.

\section{Case report}

The patient described in this report was a woman, aged 35 years, married with two children, and had a history of relapsing episodes of paroxysmal AF, for which flecainide controlledrelease was prescribed (January 2017) at the dose of $100 \mathrm{mg}$ once daily. Subsequently, due to the reappearance of AF runs during the 24-h Holter recording, associated with annoying palpitations, the patient was offered the radiofrequency ablation, which she refused. The controlled-release flecainide was therefore cancelled and replaced by the addition of bisoprolol $2.5 \mathrm{mg}$ once daily plus amiodarone $200 \mathrm{mg}$ daily. The echocardiogram (Fig. 1) was characterized by a severely depressed $(23 \%)$ left ventricular ejection fraction (LVEF) due to uniform dilatation of the left ventricle and moderate secondary mitral insufficiency. Thus, considering the echocardiographic picture compatible with the diagnosis of idiopathic dilated cardiomyopathy, the therapy was supplemented by ramipril at the dose of $5 \mathrm{mg}$ once a day, plus a mineralocorticoid receptor antagonist, i.e., canrenone $25 \mathrm{mg}$ daily, plus furosemide $25 \mathrm{mg}$ daily. In addition, due to the incumbent risk of cardioembolic events related to recurrent paroxysmal AF episodes, the patient was given rivaroxaban at the dose of $15 \mathrm{mg}$ once daily. In January 2018, despite the patient's close adherence to therapy, she started complaining about dyspnea related to minimal physical activity (New York Heart Association (NYHA) class III). The deterioration of the hemodynamic and clinical picture was related to the worsening of the functional mitral insufficiency and the occurrence of intermittent left bundle branch block. For the worsening mitral regurgitation the patient was offered a MitraClip procedure that she refused (February 2018). The echocardiogram revealed a severe dilation of the left ventricle with end-diastolic volume of $107 \mathrm{~mL}$ and end-systolic volume of $82 \mathrm{~mL}$, and severely reduced LVEF (32\%) with left atrial volume index (LAVI) of $38 \mathrm{~mL} / \mathrm{m}^{2}$. During the follow-up, in July 2018, her ventricular function was still severely reduced (LVEF $32 \%$ ) and LAVI was about $38 \mathrm{~mL} / \mathrm{m}^{2}$, despite the cardioprotective therapy. Therefore, ramipril was suspended, and after $48 \mathrm{~h}$ sacubitril/valsartan therapy was initiated, at the dosage of 24/26 mg twice daily in add-on to therapy with furosemide $25 \mathrm{mg}$ quaque altera die (qod), carvedilol $12.5 \mathrm{mg}$ twice daily, canrenoate $25 \mathrm{mg}$ once daily, and rivaroxaban $20 \mathrm{mg}$ once daily. In February 2019, sacubitril/valsartan was up titrated to 49/51 mg/bis in die (bid). In May 2019, sacubitril/ valsartan was up titrated to $97 / 103 \mathrm{mg} / \mathrm{bid}$, but patient had symptomatic hypotension, so the dosing was reduced to $49 / 51$ $\mathrm{mg} / \mathrm{bid}$. At the subsequent follow-up visit, sinus rhythm was restored and LVEF progressively recovered, with a marked reduction of chamber dimensions and cardiac volumes. In May 2019 , LVEF was $35 \%$, and LAVI was reduced to $30 \mathrm{~mL} / \mathrm{m}^{2}$. At present, the patient is in good conditions and furosemide dosing has been curtailed to the $25 \mathrm{mg}$ qod (Fig. 2).

The patient whose clinical description constitutes the subject of the present article, gave informed consent to participate in the study, and provided informed consent for publication. 

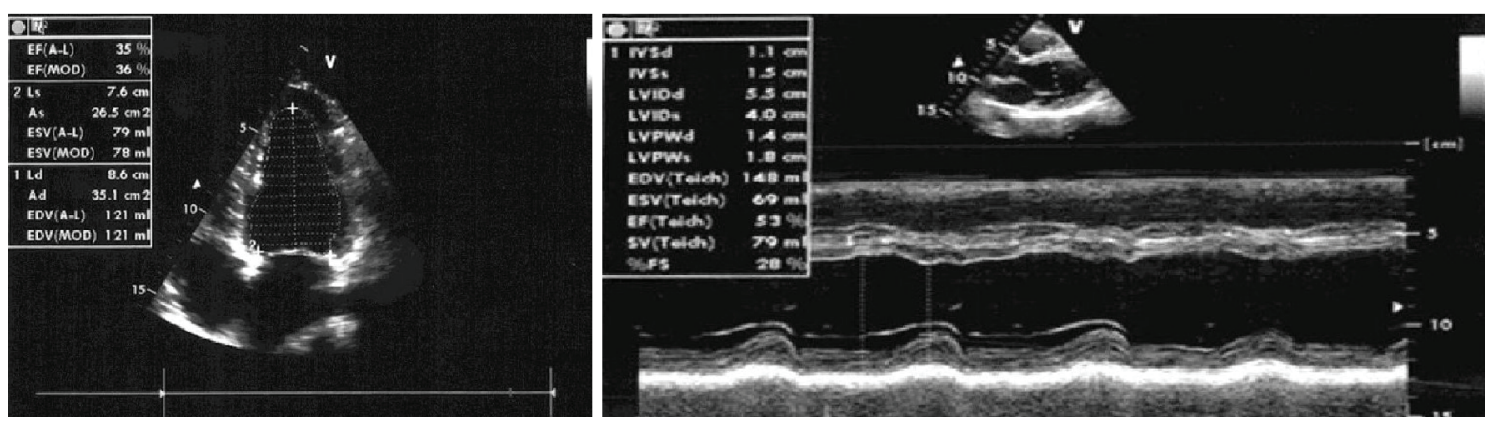

Figure 2. Echocardiography after 10 months of therapy with sacubitril/valsartan.

\section{Discussion}

The case illustrated is an example of the efficacious action of sacubitril/valsartan which in a 20-month follow-up has shown to favorably modify the pump efficiency, reduce congestion, antagonize atrial electroanatomical remodeling, thereby favoring the retrieval of the sinus rhythm. In reality, the judgment cannot be generalized to all the decompensated patients since the present study is a case report and not a RCT study. However, our experience, albeit limited, with sacubitril/valsartan used as an upstream therapy for AF leads us to assert that the drug could play a role in preventing AF recurrences through its multiple pharmacodynamic actions, including the antagonism against the renin-angiotensin-aldosterone system (RAAS), the reduction of the ventricles' hemodynamic overload, the improvement of aspiration of venous caval and pulmonary venous blood respectively by the right atrium and the left atrium thanks to the favorable modulation of atrial compliance by the ANP, whose half-life is increased by sacubitril/ valsartan.

\section{Conclusions}

Upstream therapy of AF, despite the mixed results reported so far, could have sacubitril/valsartan as its ideal drug, because this molecule acts in a balanced way on the electroanatomic remodeling of the atria thanks to the amplification of the beneficial role of the ANF obtained by the inhibition of the neprilysin which is responsible for its degradation.

The drug, favoring the improvement of the atrial reservoir phase, is able to harmoniously regulate the atrial filling during the systole. The improvement of the mechanical properties of the atrium is then mirrored by an improvement of its electrophysiological properties. Therefore, there is either a greater regularity of the ventricular response or even a restoration of the sinus rhythm, as in the present case of AF converted after the introduction in the therapy of sacubitril valsartan.

\section{Acknowledgments}

None to declare.

\section{Financial Disclosure}

The authors certify that no funding has been received for the conduct of this study. Novartis Farma SpA, Italy, provided unconditional support for the publication of the manuscript.

\section{Conflict of Interest}

The authors Renato De Vecchis, Andrea Paccone and Marco Di Maio have no conflicts of interest that are directly relevant to the content of this study.

\section{Author Contributions}

RDV and AP contributed to conceptualization; RDV contributed to data curation; RDV, AP and MDM contributed to writing and original draft preparation; RDV, AP and MDM contributed to writing, review and editing.

\section{Data Availability}

The data supporting the findings of this study are available from the corresponding author upon reasonable request.

\section{References}

1. Wijffels MC, Kirchhof CJ, Dorland R, Allessie MA. Atrial fibrillation begets atrial fibrillation. A study in awake chronically instrumented goats. Circulation. 1995;92(7):1954-1968.

2. Allessie M, Ausma J, Schotten U. Electrical, contractile and structural remodeling during atrial fibrillation. Cardiovasc Res. 2002;54(2):230-246.

3. Daoud EG, Marcovitz P, Knight BP, Goyal R, Man KC, Strickberger SA, Armstrong WF, et al. Short-term effect of atrial fibrillation on atrial contractile function in humans. Circulation. 1999;99(23):3024-3027.

4. Nattel S, Li D. Ionic remodeling in the heart: pathophysiological significance and new therapeutic opportunities for atrial fibrillation. Circ Res. 2000;87(6):440-447. 
5. Shi Y, Li D, Tardif JC, Nattel S. Enalapril effects on atrial remodeling and atrial fibrillation in experimental congestive heart failure. Cardiovasc Res. 2002;54(2):456-461.

6. Kumagai K, Nakashima H, Urata H, Gondo N, Arakawa $\mathrm{K}$, Saku K. Effects of angiotensin II type 1 receptor antagonist on electrical and structural remodeling in atrial fibrillation. J Am Coll Cardiol. 2003;41(12):2197-2204.

7. Li D, Shinagawa K, Pang L, Leung TK, Cardin S, Wang Z, Nattel S. Effects of angiotensin-converting enzyme inhibition on the development of the atrial fibrillation substrate in dogs with ventricular tachypacing-induced congestive heart failure. Circulation. 2001;104(21):26082614.

8. Disertori M, Quintarelli S. Renin-angiotensin system and atrial fibrillation: understanding the connection. J Atr Fibrillation. 2011;2:1-9.

9. Belluzzi F, Sernesi L, Preti P, Salinaro F, Fonte ML, Perlini S. Prevention of recurrent lone atrial fibrillation by the angiotensin-II converting enzyme inhibitor ramipril in normotensive patients. J Am Coll Cardiol. 2009;53(1):2429.

10. Madrid AH, Bueno MG, Rebollo JM, Marin I, Pena G, Bernal E, Rodriguez A, et al. Use of irbesartan to maintain sinus rhythm in patients with long-lasting persistent atrial fibrillation: a prospective and randomized study. Circulation. 2002;106(3):331-336.

11. Savelieva I, Kakouros N, Kourliouros A, Camm AJ. Upstream therapies for management of atrial fibrillation: review of clinical evidence and implications for European Society of Cardiology guidelines. Part II: secondary prevention. Europace. 2011;13(5):610-625.

12. GISSI-AF Investigators, Disertori M, Latini R, Barlera S, Franzosi MG, Staszewsky L, Maggioni AP, et al. Valsartan for prevention of recurrent atrial fibrillation. N Engl J Med. 2009;360(16):1606-1617.

13. ACTIVE I Investigators, Yusuf S, Healey JS, Pogue J, Chrolavicius S, Flather M, Hart RG, et al. Irbesartan in patients with atrial fibrillation. N Engl J Med. 2011;364(10):928-938.

14. Goette A, Schon N, Kirchhof P, Breithardt G, Fetsch T, Hausler KG, Klein HU, et al. Angiotensin II-antagonist in paroxysmal atrial fibrillation (ANTIPAF) trial. Circ Arrhythm Electrophysiol. 2012;5(1):43-51.

15. Disertori M, Barlera S, Staszewsky L, Latini R, Quintarelli S, Franzosi MG. Systematic review and meta-analysis: renin-Angiotensin system inhibitors in the prevention of atrial fibrillation recurrences: an unfulfilled hope. Cardiovasc Drugs Ther. 2012;26(1):47-54.

16. Dorian P, Singh BN. Upstream therapies to prevent atrial fibrillation. Eur Heart J Suppl. 2008;10(Suppl H):H11H31.

17. Kumagai K, Nakashima H, Saku K. The HMG-CoA reductase inhibitor atorvastatin prevents atrial fibrillation by inhibiting inflammation in a canine sterile pericarditis model. Cardiovasc Res. 2004;62(1):105-111.

18. Shiroshita-Takeshita A, Brundel BJ, Burstein B, Leung TK, Mitamura H, Ogawa S, Nattel S. Effects of simvastatin on the development of the atrial fibrillation substrate in dogs with congestive heart failure. Cardiovasc Res.
2007;74(1):75-84.

19. Young-Xu Y, Jabbour S, Goldberg R, Blatt CM, Graboys $\mathrm{T}$, Bilchik B, Ravid S. Usefulness of statin drugs in protecting against atrial fibrillation in patients with coronary artery disease. Am J Cardiol. 2003;92(12):1379-1383.

20. Tveit A, Grundtvig M, Gundersen T, Vanberg P, Semb AG, Holt E, Gullestad L. Analysis of pravastatin to prevent recurrence of atrial fibrillation after electrical cardioversion. Am J Cardiol. 2004;93(6):780-782.

21. Maggioni AP, Fabbri G, Lucci D, Marchioli R, Franzosi MG, Latini R, Nicolosi GL, et al. Effects of rosuvastatin on atrial fibrillation occurrence: ancillary results of the GISSI-HF trial. Eur Heart J. 2009;30(19):2327-2336.

22. Chen WT, Krishnan GM, Sood N, Kluger J, Coleman CI. Effect of statins on atrial fibrillation after cardiac surgery: a duration- and dose-response meta-analysis. J Thorac Cardiovasc Surg. 2010;140(2):364-372.

23. Suleiman M, Koestler C, Lerman A, Lopez-Jimenez F, Herges R, Hodge D, Bradley D, et al. Atorvastatin for prevention of atrial fibrillation recurrence following pulmonary vein isolation: a double-blind, placebo-controlled, randomized trial. Heart Rhythm. 2012;9(2):172-178.

24. Rahimi K, Emberson J, McGale P, Majoni W, Merhi A, Asselbergs FW, Krane V, et al. Effect of statins on atrial fibrillation: collaborative meta-analysis of published and unpublished evidence from randomised controlled trials. BMJ. 2011;342:d1250.

25. Mozaffarian D, Psaty BM, Rimm EB, Lemaitre RN, Burke GL, Lyles MF, Lefkowitz D, et al. Fish intake and risk of incident atrial fibrillation. Circulation. 2004;110(4):368373.

26. Frost L, Vestergaard P. n-3 Fatty acids consumed from fish and risk of atrial fibrillation or flutter: the Danish Diet, Cancer, and Health Study. Am J Clin Nutr. 2005;81(1):50-54.

27. Brouwer IA, Heeringa J, Geleijnse JM, Zock PL, Witteman JC. Intake of very long-chain n-3 fatty acids from fish and incidence of atrial fibrillation. The Rotterdam Study. Am Heart J. 2006;151(4):857-862.

28. Nodari S, Triggiani M, Campia U, Manerba A, Milesi G, Cesana BM, Gheorghiade M, et al. n-3 polyunsaturated fatty acids in the prevention of atrial fibrillation recurrences after electrical cardioversion: a prospective, randomized study. Circulation. 2011;124(10):1100-1106.

29. Kowey PR, Reiffel JA, Ellenbogen KA, Naccarelli GV, Pratt CM. Efficacy and safety of prescription omega-3 fatty acids for the prevention of recurrent symptomatic atrial fibrillation: a randomized controlled trial. JAMA. 2010;304(21):2363-2372.

30. Bianconi L, Calo L, Mennuni M, Santini L, Morosetti P, Azzolini P, Barbato G, et al. n-3 polyunsaturated fatty acids for the prevention of arrhythmia recurrence after electrical cardioversion of chronic persistent atrial fibrillation: a randomized, double-blind, multicentre study. Europace. 2011;13(2):174-181.

31. Liu T, Korantzopoulos P, Shehata M, Li G, Wang X, Kaul S. Prevention of atrial fibrillation with omega-3 fatty acids: a meta-analysis of randomised clinical trials. Heart. 2011;97(13):1034-1040. 
32. Mozaffarian D, Marchioli R, Macchia A, Silletta MG, Ferrazzi P, Gardner TJ, Latini R, et al. Fish oil and postoperative atrial fibrillation: the Omega-3 Fatty Acids for Prevention of Post-operative Atrial Fibrillation (OPERA) randomized trial. JAMA. 2012;308(19):2001-2011.

33. Watson CJ, Phelan D, Xu M, Collier P, Neary R, Smolenski A, Ledwidge M, et al. Mechanical stretch up-regulates the B-type natriuretic peptide system in human cardiac fibroblasts: a possible defense against transforming growth factor-beta mediated fibrosis. Fibrogenesis Tissue Repair. 2012;5(1):9.

34. De Vecchis R, Paccone A, Di Maio M. Favorable effects of sacubitril/valsartan on the peak atrial longitudinal strain in patients with chronic heart failure and a history of one or more episodes of atrial fibrillation: a retrospective cohort study. J Clin Med Res. 2020;12(2):100-107.

35. De Vecchis R, Paccone A, Di Maio M. Sacubitril/Valsartan Therapy for 14 Months Induces a Marked Improvement of Global Longitudinal Strain in Patients With
Chronic Heart Failure: A Retrospective Cohort Study. Cardiol Res. 2019;10(5):293-302.

36. Martens P, Nuyens D, Rivero-Ayerza M, Van Herendael H, Vercammen J, Ceyssens W, Luwel E, et al. Sacubitril/valsartan reduces ventricular arrhythmias in parallel with left ventricular reverse remodeling in heart failure with reduced ejection fraction. Clin Res Cardiol. 2019;108(10):1074-1082.

37. Russo V, Bottino R, Rago A, Papa AA, Liccardo B, Proietti R, Manna V, et al. The effect of sacubitril/valsartan on device detected arrhythmias and electrical parameters among dilated cardiomyopathy patients with reduced ejection fraction and implantable cardioverter defibrillator. J Clin Med. 2020;9(4):1111.

38. Suo Y, Yuan M, Li H, Zhang Y, Li Y, Fu H, Han F, et al. Sacubitril/valsartan improves left atrial and left atrial appendage function in patients with atrial fibrillation and in pressure overload-induced mice. Front Pharmacol. $2019 ; 10: 1285$. 